\title{
Sophisticated synthesis of monosubstituted piperazines - from a batch reaction vessel to a flow (microwave) reactor ${ }^{+}$
}

\author{
Dana Němečková ${ }^{1}$, Eva Havránkováa ${ }^{1}$ Jan Šimbera ${ }^{1}$, Richard Ševčík ${ }^{1, *}$ and Pavel Pazdera ${ }^{1}$ \\ 1 Department of Chemistry, Masaryk university, Kotlářská 2, Brno, 611 37, Czech Republic \\ * Correspondence: ja_richard@email.cz; Tel.: +420-54949-2677 \\ + Presented at the title, place, and date.
}

Received: date; Accepted: date; Published: date

\begin{abstract}
We reported a novel sophisticated synthetic method for preparation of monosubstituted piperazine derivatives, which can now be easily prepared in a one-pot-one-step way from a free piperazine with no need of introduction of a protecting group bringing further disadvantages in terms of time, costs and reduced yields. Reactions proceeding either at room or higher temperatures in common solvents employ with advantage heterogeneous catalysis by metal ions supported on commercial polymeric resins.

Further, we have picked up a set of piperazine derivatives and studied the possibilities of microwave acceleration of given synthetic reactions. Our investigation proceeded from a simple batch technique to the construction of a flow microwave reactor and resulted in promising findings which are summarized and discussed in our contribution.
\end{abstract}

Keywords: piperazine; monosubstituted; heterocyclic; catalysis; heterogeneous; microwave; synthesis, supported catalyst; reactor

\section{Introduction}

Generally, piperazine derivatives are very desirable building blocks for the production of pharmaceuticals and they have a great potential in the field of pharmaceutical research and development [1-4]. However, their broader use in the pharmaceutical industry is still limited by their high price, which is a consequence of their problematic preparation consisting of several synthetic steps including protection and deprotection of one of the piperazine nitrogen atoms. Thus, there is still a challenge for new methods to allow greater utilization of desired piperazine derivatives in pharmaceutical research and development of new drugs due to decrease of production costs for the synthesis of such building blocks.

In recent years we have published a number of reports and patents on a simplified one-step synthesis of a variety of monosubstituted piperazine derivatives [5-7] which are now to be offered for a commercial sale. Although a simplified synthetic method for piperazine derivatives was published, still we studied intensively ways to accelerate and further simplify described syntheses especially with regard to a potential commercial manufacturing in a larger scale. We focused on utilization of microwave energy and preliminary experiments involving a batch technique showed promising results. But we continued further and especially for a large-scale experiment we proposed and verified an idea of a flow reactor equipped with a microwave unit. Obtained results and comparisons are discussed in the article. 


\section{Materials and Methods}

Synthetic procedures of a classic scheme are described in the patents [5-7]. Batch experiments involving microwave energy follow classic procedures, but microwave irradiation was employed instead of regular heating. Crude products were then isolated and purified (if necessary) in the same way as described for the classic methods. In case of a flow technique, processes were modified as follows. At first a solution of piperazine or piperazine monohydrochloride (in situ from piperazine and piperazine dihydrochloride hydrate) was prepared according to a classic procedure and poured into a reservoir flask of a flow reactor. Then a catalyst was loaded into a reaction flask placed in a microwave oven (a detailed scheme is described on Fig. 1). Then pump was switched on to run the flow slowly. A corresponding reagent was added portion wise into the reserve flask to be introduced into the reaction mxture. Microwave oven power was always (batch and flow experiments) set to a minimal energy (10 $\%$ of a maximal power, i.e. $80 \mathrm{~W}$ ) and applied using a pulse mode (typically $3 \mathrm{sec}$. of a set power then 4 sec. with no power) to keep a reaction mixture in the reaction flask slightly boiling. Products were routinely identified using ${ }^{1} \mathrm{H}$ and ${ }^{13} \mathrm{C}-\mathrm{NMR}$ and melting point determination.

FT-IR spectra were measured on Bruker ATR Diamond. NMR spectra were obtained on a Bruker Avance NMR III TM 300/75 MHz for hydrogen/carbon spectrum, using wideband probe BBFO. Melting points were measured on a Boetius apparatus PHMK 05 (VEB Kombinat Nagema) with temperature gradient of $4{ }^{\circ} \mathrm{C} / \mathrm{min}$ and their values are uncorrected. The reactions were monitored by TLC, which was conducted on aluminum plates TLC Silica gel 60 F254 by Merck. The detection methods and the eluents used for particular compounds are given in the procedure protocols for corresponding syntheses of the compounds [5-7]. Generally, the presence of substances was detected either by using UV lamp CAMAG (wavelength $254 \mathrm{~nm}$ or $366 \mathrm{~nm}$ ) for compounds absorbing at the wavelengths, iodine vapors, or solution of ninhydrin (solution containing $2 \mathrm{~g}$ of ninhydrin, $100 \mathrm{~mL}$ of $\mathrm{n}$-butanol and $20 \mathrm{~mL}$ of acetic acid) when $\mathrm{NH}_{2}$ and $\mathrm{NH}$ groups were present in the detected molecule.

\section{Results and Discussions}

As it was described in patented procedures [5-7], we discovered and verified a new and simple one-pot-one-step method for synthesis of a variety of monosubstituted piperazine derivatives which can now be prepared in high yields and purity while keeping low costs. Moreover, compounds are now commercially available for research and development via www.entwickchemicals.com and www.fichema.cz webpages.

The principle of the synthetic method is based on a reaction of a piperazine (free or as a monohydrochloride) with an appropriate reagent (mostly chloride, chloroderivative, unsaturated compound etc.) which is catalyzed by metal ions supported on a polymeric resin $[8,9]$. Reactions proceed in a common solvent (usually methanol or acetic acid for mono-acylation and similar reactions) at room temperature or under reflux. Details of the preparations can be found in patents or literature [5-7].

Since the most of the reactions proceed under reflux for several hours, we were searching for other ways to shorten especially reflux time of the reactions so as to bring further savings in energy consumption, cooling and time. Thus we focused on a microwave energy for heating and first of all we set up batch experiments using a commercially available microwave oven which was only slightly modified as a small hole for the usage of a condenser or for input/ output hosepipe has to be bored into the top wall of the oven. A series of reactions was selected for the comparison of different synthetic procedures - classic flask procedure (denoted as A), microwave batch process (B) and process employing a flow microwave reactor ( C).

In case of setup B, a reaction flask containing prepared reaction mixture and a catalyst was placed into the oven and a glass tube was attached to it passing through the hole in the top wall of the oven. 
Then a back condenser was mounted on the top of the glass tube to be out of the reach of microwaves.

The first setup of a microwave flow reactor prototype (procedure C) which was used for all of the microwave assisted experiments (batch and flow setup) is presented on Figure 1. When employing

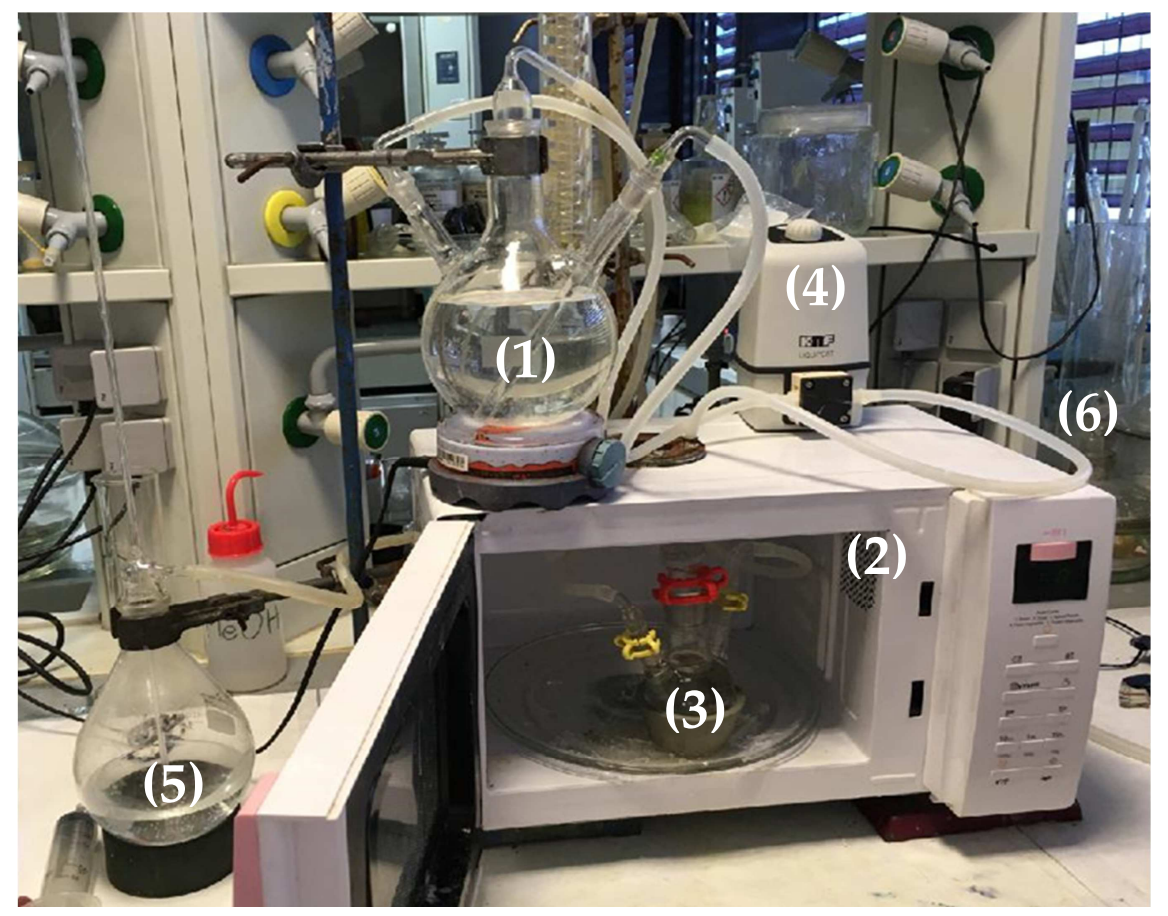

Figure 1. Functional prototype of a flow microwave reactor used for the experiments.

a flow reactor, a piperazine solution (appropriate form) is first poured into a reservoir flask (1) on the top of the oven (2), then a small reaction flask (3) is put into the oven and a catalyst is placed into it (with advantage closed in a catalytic bed). Input and output hosepipe connecting a reaction flask is passed through holes in the top wall of the oven. Then the pump of a membrane or peristaltic type (4) is switched on and the entire system (hosepipe, pump and the reaction flask) is filled with a reaction mixture. Then the second starting compound is added portion wise into the circulating reaction mixture in the reservoir flask. A reservoir flask is then attached to a safety flask (5) balancing the pressure fluctuation in the circle. All of the parts are linked together with a hosepipe made of silicone (6) and they constitute a closed system. Then microwave oven is to be switched on to initiate the reaction. However, the flow reactor can be used only in a flow mode when microwave heating is not required and a reaction proceeds only when a solution is passing through catalytic bed. Similarly, only microwave heating without catalytic bed can be used in case of uncatalyzed reactions. When using a microwave oven, the microwave power was always decreased (typically to $10 \%$ of a maximum power) and applied using a pulse mode to keep a reaction mixture only slightly boiling. The microwave irradiation was finished when the conversion of the reaction was full or highest as it was monitored by TLC.

For the comparison of a series of synthetic reactions yielding monosubstituted piperazine derivatives was selected. Excepting reaction of piperazine with methyl chlorocarbonate, reactions follow Scheme 1 starting from piperazine monohydrochloride and proceed in methanol under reflux for several hours. A number of experiments when varying molar ratios, catalyst or other variables was performed and the best results of all of the experiments employing different synthetic techniques are then summarized in Table 1. 

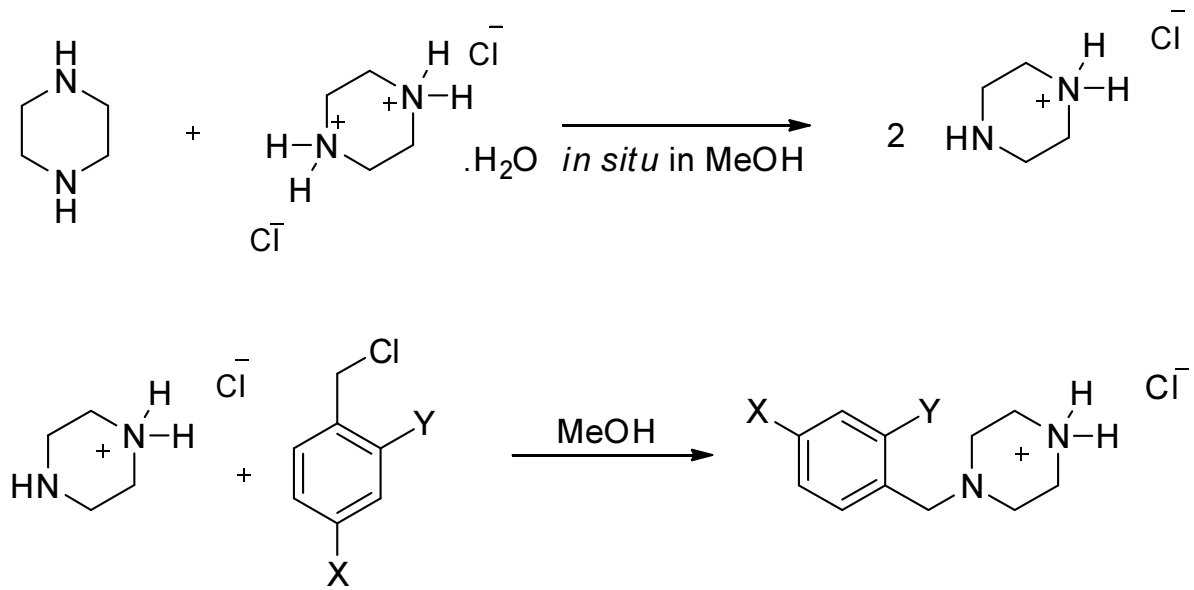

Scheme 1. A general reaction scheme of monosubstituted piperazine derivatives synthesis - model reaction of substituted benzyl derivatives.

Table 1. Results of syntheses of monosubstituted piperazine derivatives using different synthetic techniques.

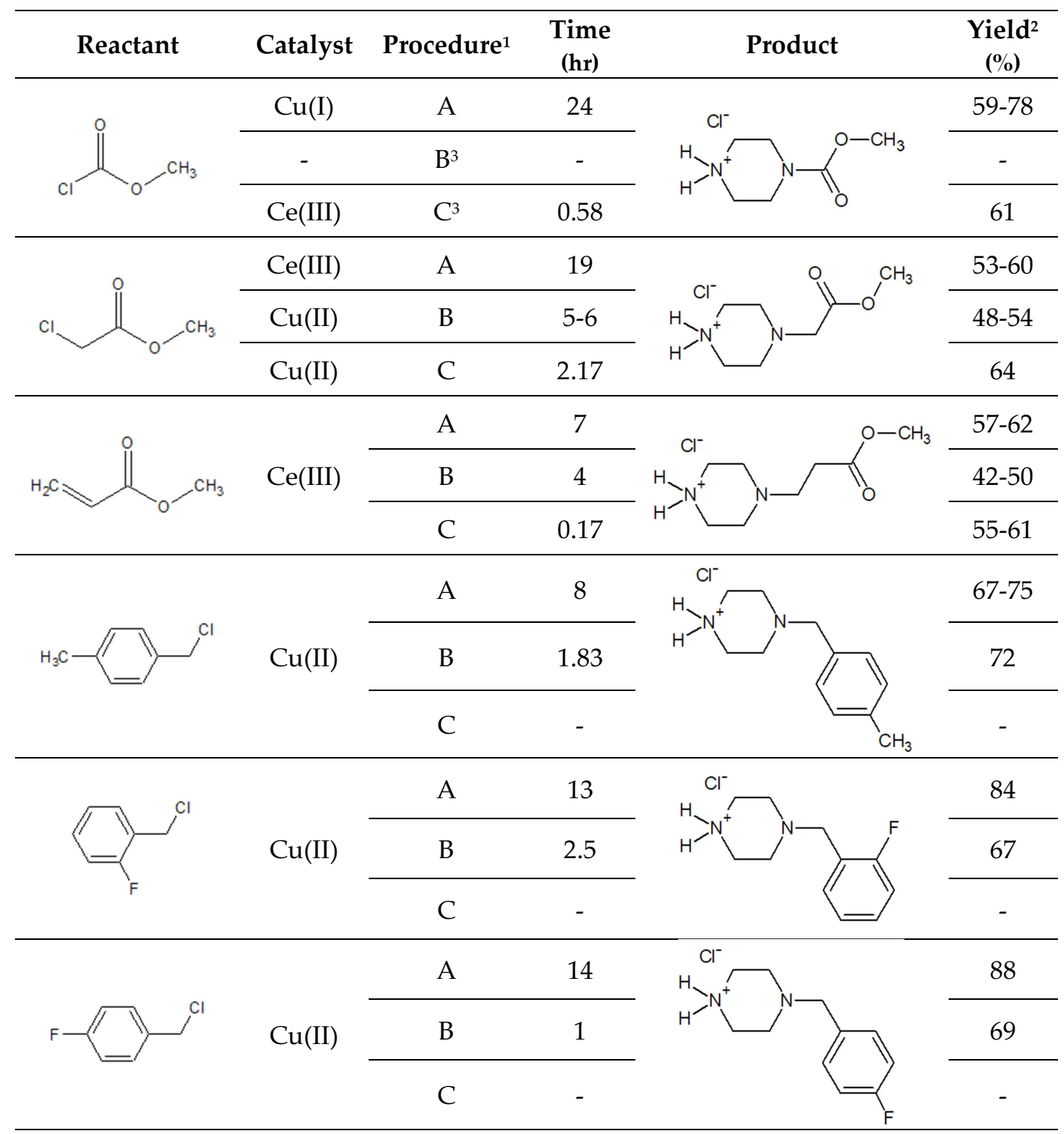

${ }^{1}$ Proc. A were performed according to patents [5-7], proc. B (batch) and C (flow) proceed under MW irradiation.

${ }^{2}$ Yield of a recrystallized product with respect to piperazine monohydrochloride or free piperazine.

${ }^{3}$ Reaction proceeds at room temperature and thus only a simple flow mode using catalyst was applied. 
Obtained results confirmed our expectation as it can be clearly seen that procedures proceeding under microwave irradiation give the same results concerning yields (and purity) but reactions are in advantage finished in considerable shortened times, mostly in a several times lower period. Especially in case of reactions where all of the three techniques were studied it is obvious that reaction time is significantly decreased when changing from classic flask method through a batch process to a flow microwave reactor processing. The reason can be found in an active part of the flow system - a small reaction flask in the oven. A reaction flask (of 100-150 ml volume) is always filled up with only a portion of a reaction solution which is influenced by a relatively high amount of a catalyst placed in a catalytic bed and thus reaction can proceed very quickly. A catalyst : reactants ratio can be then even 10 times higher in case of a flow process than in batch and classic processes and amount of a catalyst can be in fact independent on a volume of a reaction mixture. Additionally, a small volume of a reaction mixture which is exposed to microwaves then requires only a low microwave power, i.e. a commercially available microwave oven instead of a huge and expensive instrument for large volumes.

Nonetheless some problems may occur when some intermediate or a by-product start to crystalize from the solution. Then it can be deposited somewhere in the circle or it can cause a pump malfunction. Although a small quantity of a solid in a flowing mixture is not supposed to be an issue. Then optionally a solid particle filter should be placed into the circle to prevent leakage of solids from a reaction flask. Of course, a problem may remain when a large quantity of a solid intermediate/ by-product is formed and prevail in the mixture, then the flow system may collapse in spite of a filter employed as solid mass may form a stopper in the reaction flask to stop a running flow. For this type of reactions employment of a flow reactor is not recommended.

In a consequence with performed experiments we focused on the design of a commercially available reactor reflecting obtained results and fixing observed issues. In cooperation with a private company we introduced and patented a utility model of a flow reactor with a microwave unit and/or catalytic bed [10] as it is presented on Figure 2.

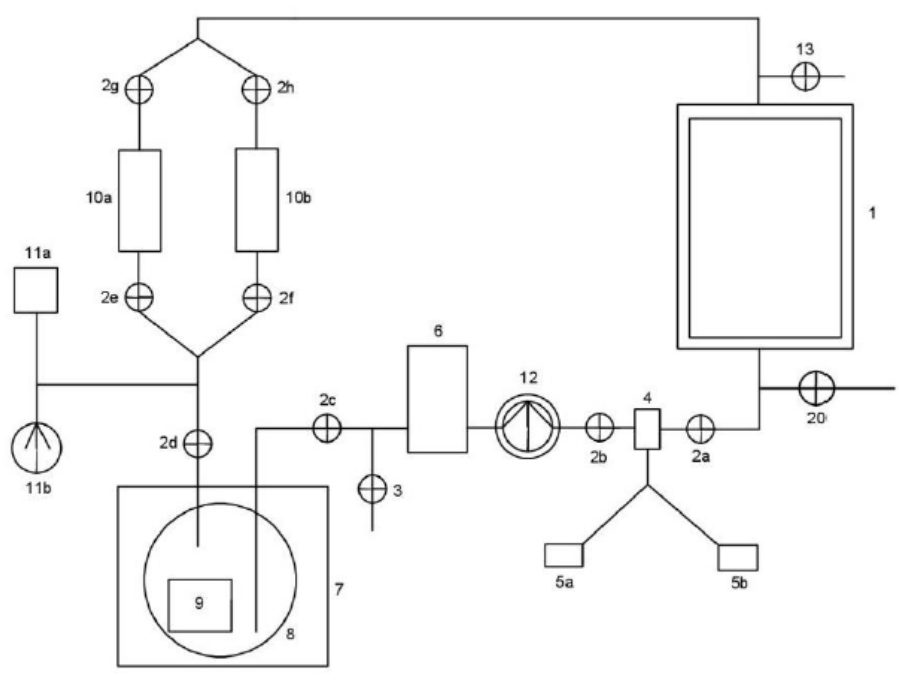

(a)

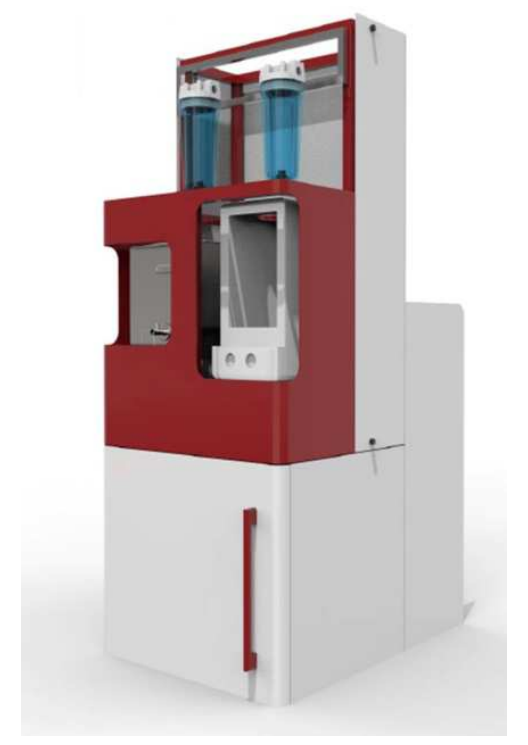

(b)

Figure 2. Patented principle of a flow reactor with microwave unit and/or catalytic bed. (a) Scheme of a flow microwave/catalytic bed reactor system; (b) Draft of an outer design of a flow reactor.

A flow reactor can be used in three working modes: i) microwave only mode for employment of polar or polarizable reagents, ii) microwave mode with catalytic bed when synergy of both of the processes is necessary and iii) catalytic bed only mode when reaction proceeds at room temperature and no other acceleration is needed. A proposed design is variable and allows to be adjusted for a 
variety of volumes. Moreover, it can be combined with an ultrasonic flow reactor which was presented recently and described in patent [11]. Nowadays we are searching for a partner to finish design and verify functionality of a flow microwave reactor prototype, hopefully, to be offered for a commercial use.

\section{Conclusion}

In the article we summarize the process of our research of monosubstituted piperazine derivatives passing from the invention of a new simple one-step method of their synthesis to a flow microwave reactor which enables piperazine derivatives manufacturing more effectively in larger scale with prospect to their potential manufacturing. No need to add that a flow microwave reactor equipped with a catalytic bed can be employed for synthesis of a variety of different products as it can work in three working modes depending on a type of reaction. Thus, it can be interesting for a wider sphere of chemists and manufacturers.

On the basis of a number of experiments, we showed that a new and simple method of synthesis of monosubstituted piperazine derivatives can be further accelerated and can be made more efficient using a microwave irradiation. Because a batch microwave process can be considered as a low cost and simple process not requiring huge and expensive microwave device only when a small volume of a reaction mixture is involved, we proceeded to an idea of a flow reactor consisting of a circulating system with only a small active part which uses a small commercially available microwave unit. Such a design brings a lot of benefits such as variability, speed and efficiency for a low price as it can be constructed using cheap and easily available parts and materials.

\section{Patents}

Procedures for a direct $N$-monosubstitution of piperazine are protected under patent numbers CZ 305854 (2016), CZ 305317 (2015), CZ 304520 (2014). Description of supported metal catalysts can be found under patent numbers CZ 305277 (2015) and CZ 303987 (2013). Technology of a batch microwave process and of a flow microwave process including a flow reactor is protected as a classified know-how of Masaryk University. A flow reactor with microwave unit and/or catalytic bed is protected as a utility model under number UV 32201 (2018). A flow ultrasonic reactor is protected as a utility model under number UV 24590 (2012).

Author Contributions: chemical experiments, analysis, research, D.N.; batch and flow microwave assisted experiments, E.H. and J.Š.; research, reactor design, writing-original draft preparation, R.Š.; conceptualization, reactor design, review and editing P.P.

Funding: This research was supported by internal grant of Masaryk University number MUNI/31/53569/2016 (Proof of Concept) which was funded by GAMA program of Technology Agency of the Czech Republic (grant number TG02010067).

Conflicts of Interest: The authors declare no conflict of interest.

\section{References}

1. Al-Ghorbani, M.; Bushra A.B.; Zabiulla, S.; Mamatha, S. V.; Khanum, S.A. Piperazine and Morpholine: Synthetic Preview and Pharmaceutical Applications. J. Chem. Pharm. Res. 2015, 7(5), 281-301, DOI: 10.5958/0974-360X.2015.00100.6. 
2. Singh, K; Siddiqui, H.H.; Shakya, P.; Bagga, P.; Kumar, A., Khalid, M.; Arif, M.; Alok, S. Piperazine - A Biologically Active Scaffold. Int. J. Pharm. Sci. Res. 2015, 6(10), 4145-58. DOI: 10.13040/IJPSR.0975-8232.6(10).

3. Chen, L.; Chen, H.; Chen, P.; Zhang, W.; Wu, Ch.; Sun, Ch.; Luo, W.; Zheng, L.; Liu, Z.; Liang, G. Development of 2-amino-4-phenylthiazole analogues to disrupt myeloid differentiation factor 88 and prevent inflammatory responses in acute lung injury. Eur. J. Med. Chem. 2019, 161, 22-38, DOI: 10.1016/j.ejmech.2018.09.068.

4. Szczepańska, K. et al. Synthesis and biological activity of novel tert-butyl and tert-pentylphenoxyalkyl piperazine derivatives as histamine H3R ligands. Eur. J. Med. Chem. 2018, 152, 223-234, DOI: 10.1016/j.ejmech.2018.04.043.

5. Pazdera, P.; Zberovská, B.; Němečková, D. (2014). Czech patent No. CZ 304520. IPO Prague, Czech Republic.

6. Pazdera, P.; Zberovská, B.; Herová, D. (2015). Czech patent No. CZ 305317. IPO Prague, Czech Republic.

7. Pazdera, P.; Zberovská, B.; Herová, D. (2016). Czech patent No. CZ 305854. IPO Prague, Czech Republic.

8. Pazdera, P.; Zberovská, B.; Procházková, M. (2013) Czech patent No. CZ 303987. IPO Prague, Czech Republic.

9. Pazdera, P.; Zberovská, B.; Herová, D.; Datinská, V.; Šimbera, J. (2015) Czech patent No. CZ 305277. IPO Prague, Czech Republic.

10. Pazdera, P.; Němečková, D.; Havránková, E.; Šimbera, J.; Ševčík, R. (2018) Czech utility model No. CZ 32201. IPO Prague, Czech Republic.

11. Pazdera, P.; Ševčík, R. (2012) Czech utility model No. CZ 24590. IPO Prague, Czech Republic.

(C) 2019 by the authors. Submitted for possible open access publication under the terms and conditions of the Creative Commons Attribution (CC BY) license (http://creativecommons.org/licenses/by/4.0/). 